\title{
Hemiplegic shoulder pain
}

\section{Anthony B Ward}

\section{Reducing muscle tone in the subscapularis muscle with botulinum toxin can help in the treatment of shoulder pain in patients with spastic hemiplegia}

$\mathrm{S}$ houlder pain is a common problem after stroke. Its incidence is variable in up to $70 \%$ of patients ${ }^{1}$ and it often appears in the first few days. It is a marker of stroke severity, and $75 \%$ of patients complain of pain at some time in the first 12 months following a stroke. The mechanisms for the development of pain are sometimes unclear but, since the attachment of the upper limb to the trunk is muscular rather than directly skeletal, any disruption of muscular action is likely to give biomechanical problems around the shoulder, which can lead to pain. Hemiplegic shoulder pain is associated with a reduced pinch grip and shoulder shrug strength, with abnormal muscle tone, but most importantly, with sensory inattention and sensory loss. ${ }^{2}$ Patients with this problem lose movements around the shoulder and, in the context of hypertonia, a typical posture of adduction and internal rotation of the arm is seen. Physical and pharmacological treatments have had variable success and, where spasticity has occurred, the main aim has, to date, been to improve arm abduction and external rotation. The latter is a major factor in producing pain, and the paper by Yelnik and colleagues, ${ }^{3}$ in this issue of $J$ Neurol Neurosurg Psychiatry, is one of the first to demonstrate reducing the muscle tone in the subscapularis muscle to help this problem (see page 845).

Treatment for hemiplegic shoulder pain is aimed at dealing with patients' pain in the early, often flaccid, stage where the shoulder is prone to inferior subluxation, traction on the gleno-humeral capsule and soft tissue damage. Later on, troublesome spasticity may occur and movement becomes severely limited. Early intervention is thought to be advisable, as contracture can occur quite rapidly. This study is, therefore, useful in that it includes patients heading towards this complication, following which the appearance of a constrictive capsulitis occurs. The authors based their study on the hypothesis that reducing the spasticity of the main medial rotator muscle (ie, the subscapularis muscle) with botulinum toxin can help shoulder pain in patients with spastic hemiplegia, even in those with already limited range of motion, compatible with capsulitis. An improvement in the passive range of motion was expected as a parameter of the efficacy of botulinum toxin on spasticity and as a possible secondary benefit.

The mainstay of treatment is physical, but no one technique is superior over any other. Support for the limb is the most likely benefit, and the use of strapping has been advocated during the early algodystrophic phase but, later on, relieving spasticity and maintaining range of movement requires expert handling and a coordinated multidisciplinary management approach to minimise interference with rehabilitation and optimise outcome. ${ }^{4}$ Local steroid injections should be avoided unless there is clear evidence of an inflammatory lesion, but botulinum toxin injections should be considered in view of its ability to reduce pain and allow better external rotation.

Further research is needed to determine effective prophylaxis and to document the therapeutic effect of different modalities in the various presentations. The paper by Yelnik and colleagues ${ }^{3}$ is helpful in advocating the use of a safe treatment to assist the physical management of the patient and in terms of defining a strategy for prevention.

J Neurol Neurosurg Psychiatry 2007;78:789. doi: 10.1136/jnnp.2006.108803

Correspondence to: Dr Anthony B Ward, North Staffordshire Rehabil Centre, The Hywood, High Lane, Burslem, Stoke on Trent ST6 7AG, UK; anthony.ward@uhns.nhs.uk

Published Online First 14 December 2006 Competing interests: None.

\section{REFERENCES}

1 Bender L, McKenna K. Hemiplegic shoulder pain: defining the problem and its management. Disabil Rehabil 2001;23:698-705.

2 Diamond PT. Rehabilitative management of poststroke visuospatial inattention. Disabil Rehabil 2001;23:407-12.

3 Yelnik AP, Colle FM, Bonan IV, et al. Treatment of shoulder pain in spastic hemiplegia by reducing spasticity of the subscapularis muscle: a randomised, double blind, placebo controlled study of botulinum toxin type A. J Neurol Neurosurg Psychiatry 2007;78:845-8.

4 Turner-Stokes L, Jackson D. Shoulder pain after stroke: a review of the evidence base to inform the development of an integrated care pathway. Clin Rehabil 2002;16:276-98. 